\title{
MACHINE LEARNING BASED PREDICTIVE MODEL FOR SURFACE ROUGHNESS IN CYLINDRICAL GRINDING OF AL BASED METAL MATRIX COMPOSITE
}

\author{
Ferhat $\mathrm{UCAR}^{I^{*}}$, Nida KATI ${ }^{2}$
}

\begin{abstract}
The Metal Matrix Composite (MMC) technology of today is a challenging topic with novel developments. MMC materials have a key role in space, automotive, naval, and aviation industries and supplies of the defense industry owing to their superior specifications. Hence, advancing the machining quality of these materials is an essential point. This work presents a machine learning based prediction model for the surface roughness of LM25/SiC/4 composite. The related dataset is linked to an MMC, which is machined with a cylindrical grinder, so the input parameters of the model are depth of cut, wheel velocity, feed, and velocity of the work piece. The proposed model is based on a state of the art machine-learning method called Gaussian Process Regression (GPR). Alongside its robust performance in the small datasets, GPR has the ability with its Bayesian approach basis in providing uncertainty evaluation on the predicted values. Parameter optimization is also applied to the proposed GPR model. For a better evaluation of the GPR, a support vector machine based prediction model is also tested. In addition to the data split test method, models are tested with a 5-fold cross-validation algorithm. The experimental results present that the proposed GPR model reaches an adequate accuracy in terms of $R$-square, root mean squared error, and mean absolute error criteria.
\end{abstract}

Key words: metal matrix composite, machine learning, surface roughness, GPR prediction model.

\section{Introduction}

Nowadays, the industrial fields are in a significant need for improved engineering materials, which expose high-quality specifications and efficient performance in highly critical applications. On the other hand, those advance materials should resist high temperatures, pressure, and stresses. Metal Matrix Composite (MMC) materials are the primary materials that can provide those features with their raised hardness, a low value of thermal expansion, great strength to weight ratio, durable wear resistance, and long fatigue life. For this reason, traditional materials in the fields of the automotive, aerospace, defense, and military are progressively being replaced by MMC materials [1, 2]. Amid all others, Aluminum $(\mathrm{Al})$ based MMC materials, which are reinforced by silicon carbide particles $\left(\mathrm{SiC}_{\mathrm{p}}\right)$ have quickly become one of the most preferred MMC materials of recent date [3, 4]. In the machining process

\footnotetext{
${ }^{1}$ Department of Electrical and Electronics Engineering, University of Firat, Elazig, Turkey, (fucar@ firat.edu.tr)

(D)https://orcid.org/0000-0001-9366-6124

2 Department of Metallurgy and Material Engineering, University of Firat, Elazig, Turkey, (nkati@firat.edu.tr)

(D)https://orcid.org/0000-0001-7953-1258
} 
of such exclusive materials, it is deserved a more and more advance engineering point of view to reveal perfect items to the end-users. Figure 1 shows a general block scheme which summarizes the Aluminum MMC properties, application areas and reinforcement types [2, 5].

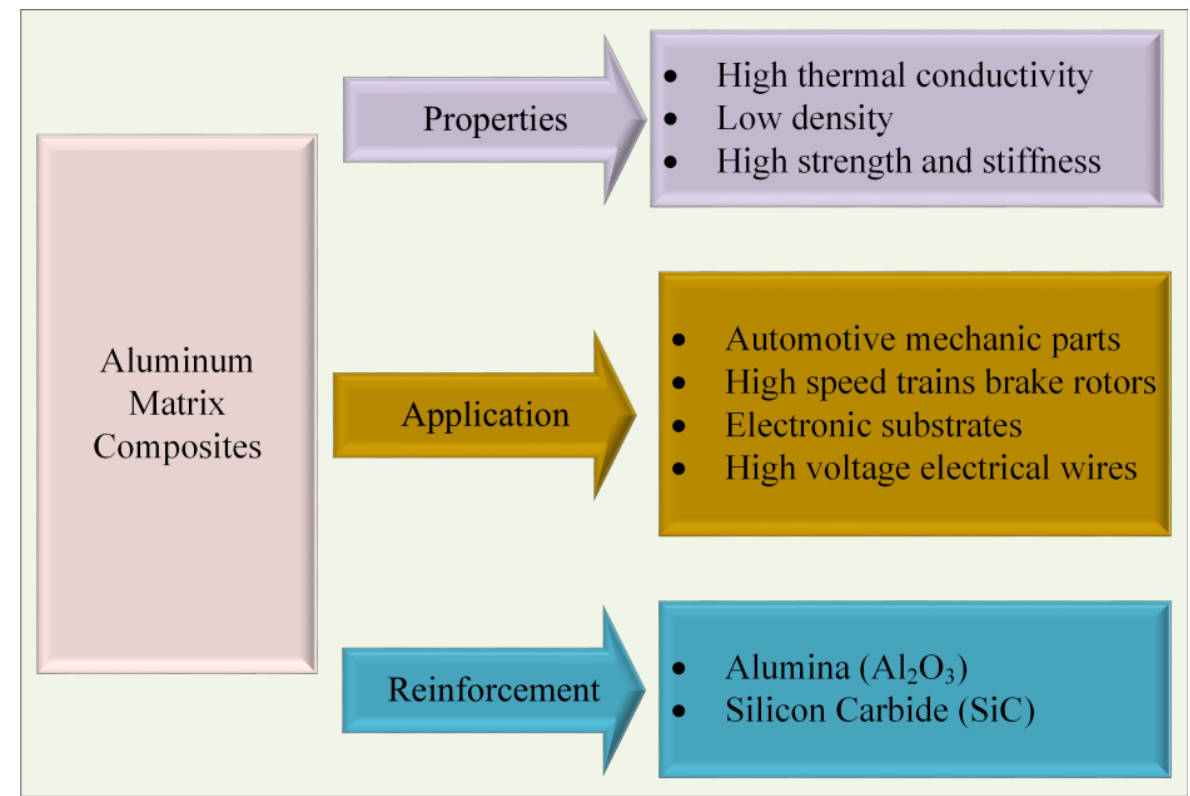

Figure 1. Aluminum MMC identification scheme.

In the modern engineering atmosphere, it is obviously reached a point that all the players of the industry increasingly have been benefit from the rapid developments of today's simulation and modeling environment. Hence, it is of great importance to use artificial intelligence capabilities in the processing of MMC materials because they have some disadvantages in processing costs and machinability [6]. While MMC materials have advanced mechanical and thermal properties, some drawbacks such as processing costs and poor machinability still stand as an obstacle in the way of broad usage of them [2]. On that account, the machinability research is an essential interest for scientists to be able to develop an intelligent production line in MMC materials. Artificial intelligence (AI) and particularly machine learning (ML) methods open the door to researchers on the way of advanced modeling of the MMC material machinability. In the last decade, there are several studies in the related literature that address AI usage in the metallurgical and materials engineering [2, 7-10]. Even in diamond machining process, ML methods stand out with their prediction abilities [11]. In [12], the authors propose an optimization model for the milling of aluminum hybrid MMC and the regression model helps to form the inputs of the MMC machinability. The authors of the study [13] propose a traditional artificial neural network (ANN) model to predict the performance parameters of an $\mathrm{Al} / \mathrm{SiC}_{\mathrm{p}} \mathrm{MMC}$ machining process and the prediction model exposes the efficient features of the process successfully. In [14], the authors present an ANN based prediction network to help the Al based MMC manufacturing process, analysis of the study cover the micro-hardness, tensile extension, yield strength, modulus, and tensile stress. In [15], the authors present an ANN model design to predict the hardness and electrical conductivity of Cu based MMC. The study [16] investigates the effect of the milling time and matrix size on an alloy fabrication, the analysis interprets the results of a traditional ANN-based prediction model. The authors of the study [17] use multiple regression analysis for estimating the machining performances of Al-based MMC, instead of using an ANN-based model and interpret the results using sophisticated mathematical models. 
In [18], the authors conduct an experimental investigation on wear properties of $\mathrm{Al} / \mathrm{SiC} \mathrm{MMC}$ and build a traditional ANN-based prediction model, the prediction target is weight loss of the specimen and the model produces an adequate performance. The input parameters of the model are production temperature, applied load force, and sliding distance.

Surface roughness is a crucial property for determining material quality. MMC with an adequate surface finish shows an improved tribological property and elegant attraction. Of course, producing the MMC with an extremely better surface finish comes with a higher cost. For that reason, scientists enounce many kinds of methods for prediction modeling and identifying the suitable parameters for surface roughness [19]. The obtained prediction models are very helpful in enhancing the surface quality in the grinding process of MMC materials because the experts in the field would assume the surface roughness without doing any real experiment and determine the input parameters according to the model results. In [20], the authors propose a prediction model (based on the traditional ANN) for surface roughness of some alloys in the end milling machine. The study [21] investigates some parameters on surface roughness in Al based MMC. To reach the results, a prediction model is designed with inputs of cutting speed, feed rate and application angle for the output as surface roughness. Another ANN based prediction model of surface roughness is proposed in [22], here the proposed model is supported with grey relational analysis to interpret the results. There are many kinds of researches, particularly based on ANN, have been conducted covering the prediction models for surface roughness analysis [23-25].

When the literature review given above is interpreted, it is seen that almost all prediction models for surface roughness estimation are based on traditional ANN structure. However, if up to date studies are examined, many applications can be found that traditional ANN structures stay behind the state of the art machine learning methods. Also, network architectures should be simple and easy to implement when dealing with real-world applications. In ANN structure, there are lots of parameters that should be optimized such as neuron numbers of hidden layers, activation function determining, and total layer numbers. ANN also has so many limitations in practice such as poor generalization performance, overfitting problem, and the possibility of local minima sticking. Thus, there is an obvious necessity to explore other artificial intelligence methods to overcome the weaknesses of the traditional ANN [26]. Meeting this requirement would provide robustness in surface roughness prediction results. Today, machine learning methods are successfully utilized for regression, classification, or clustering in materials sciences [27, 28]. Gaussian process regression (GPR) is one of the machine learning models based on the Bayesian approach and it generates a robust performance in the small datasets providing uncertainty evaluation on the predicted values [26]. With this motivation, the proposed study adopts the GPR method for modeling and prediction of surface roughness of Al-based MMC in cylindrical grinding. The dataset of the studies [3,29] is preferred because of its clear experiment results. So, the benchmark study selected from the literature for the proposed prediction model is taken into account as the results of the study [3]. The workpiece is $\mathrm{Al}-\mathrm{SiC}_{\mathrm{p}} \mathrm{MMC}$. The proposed GPR model also includes a hyperparameter optimization using the Bayesian approach. Although the GPR method is previously in use, the perfection of the GPR in the area of the surface roughness prediction researches has not been exposed. Besides, the GPR method has not been evaluated with the benchmark traditional ANN structures to deduce its reliability in surface roughness prediction. Consequently, taking cognizance of the previous theoretical conveniences provided by the GPR, the paper aims to contribute:

1. Investigation the capability of the GPR model as a novel application for predicting surface roughness using Al-based MMC cylindrical grinding open-source dataset obtained from a previously designed experimental setup; 
2. Shaping a comparative work between the proposed GPR structure and benchmark method of traditional ANN.

The layout of the article is composed as follows: Section 2 presents a concise outline of the theoretical background of the proposed GPR procedure. Section 3 defines a brief explanation of the dataset used in the prediction model. In Section 4, the experimental findings are presented alongside the GPR model experiment setup. Section 5 finalizes the article with conclusions.

\section{Gaussian Process Regression based Machine Learning Model}

Machine learning (ML) models focus on optimizing the performance of a specific task by using similar behaving instances or historical data. In general words, ML algorithms can be thought in three main topics, namely, supervised, unsupervised, and reinforcement learning structures. Since, supervised learning structure is by far the most predominant form of ML in materials science [27], we provide a brief identification about supervised learning in the following discussion before presenting the proposed machine learning algorithm.

Among ML algorithms, supervised learning is following the essentials of a similar procedure with the common fitting principle. The main aim is to find the black box (unknown) function that tries to map the known input parameters to unknown output parameters. The expected result for unknown output spaces is predicted using the extrapolation of patterns obtained in the labeled training data. Figure 2 illustrates the general workflow practice in supervised learning [27].

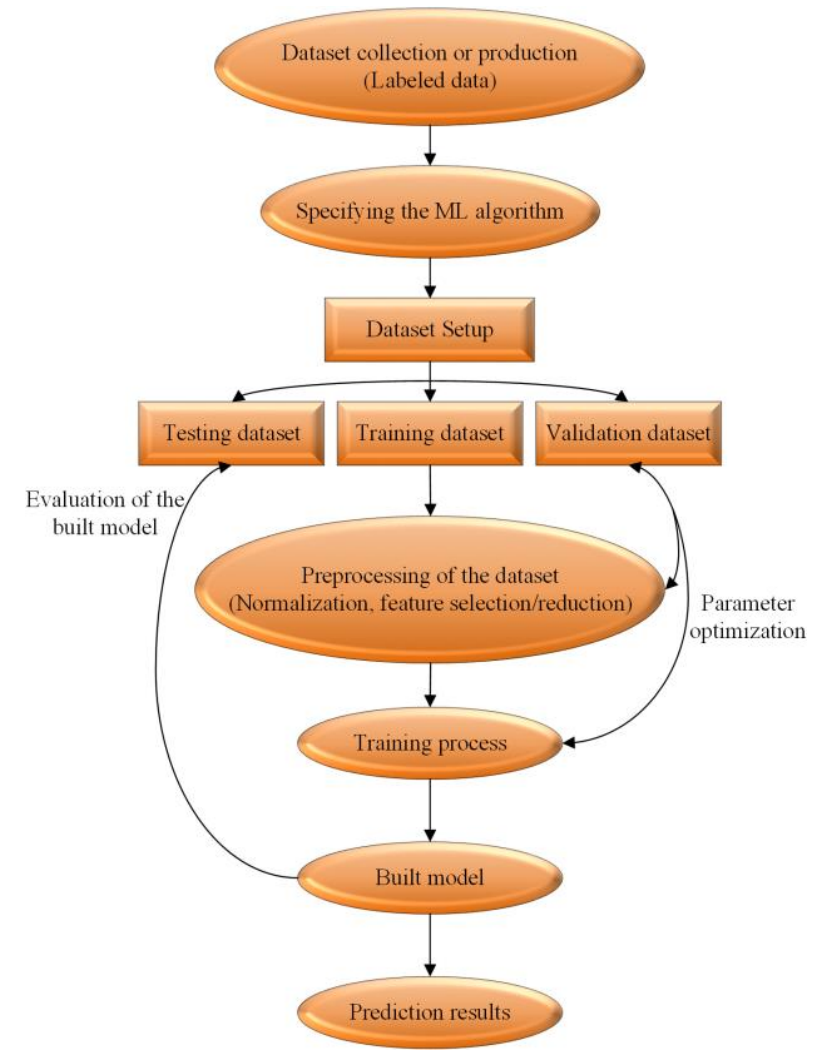

Figure 2. General workflow of the supervised learning structure.

The dataset is the backbone and the core of the learning process because the model tries to completely learn the hidden footprints of the patterns in the data distribution. When this is the case, the 
dataset collection or producing it from scratch has a key role in the whole workflow. This process is followed by the step of specifying the proper machine learning algorithm to fit the expected targets. Beyond this, dataset preparation and setup is another step in the progress. Generally, the three-part (traintest-validaiton) data split method is used for the learning process but $k$-fold crossvalidation is also used for the experiments. The data split or simple hold out method performs the model once but k-fold crossvalidation works with $\mathrm{k}$ equally sized dataset. The ML model is trained with $(k-1)$ subsets and the remaining subset is separated for testing. The procedure repeats for every single subset, iteratively [27, 30].

The other important chapter of the progress which could include normalization, feature extraction, selection, or reduction approaches is data preprocessing. Here, the data distribution would be mapped and some algorithms may translate the raw data into different feature domains to obtain the information. Now, this data could be used as the input of the algorithm. After all those processes, the ML model is ready for the training process which may also include optimization of the model parameters. No matter what, the model should not face the training data instances in the testing period. The validation set may be used in the model parameter optimization to check the cost function conditions. In the training process, ML algorithms may suffer from overfitting and underfitting. The main reason for the underfitting is generally tied to the model design lacking the capability of processing the complex data distribution or it may depend on the lack of feature presentation. In this case, the ML model produces a high training error. Conversely, a model with overfitting may perform well in the training but it fails to predict new (never seen) data, reliably. One of the ways to avoid overfitting is to track the validation error during the training instead of tracking only the training error. If the validation error stocks in a band-width value and no more decreases, we can say the ML model may face the overfitting. Before the end game which is the prediction of the outputs, the model is evaluated with the previously unseen dataset with evaluation methods aforementioned such as simple hold out or cross-validation [27].

The methodology of this paper consists of the optimized Gaussian process regression (GPR), here we present its concise picture of it. The methodological details of the well-known benchmark theories such as ANN and support vector machine (SVM) models will not be considered here. Please see the studies [2, 28] for a detailed information on those methods as well as [31, 32]. For the details of the Bayesian-based hyperparameter optimization, please see the studies [31, 32].

Gaussian process as general term consists of random variables which every single collections of those finite random variables are in the frame of a joint Gaussian distribution [26, 33]. The parameters set of a Gaussian process $g_{p}(t)$ consists of a mean function $M(t)$, and a kernel or covariance function $c\left(t, t^{\prime}\right)$ assigned to points $t$ and $t^{\prime}$. We can express those functions in (1) and (2) as:

$$
M(t)=E\left(g_{p}(t)\right)
$$

$$
\operatorname{Cov}\left(g_{p}(t), g_{p}\left(t^{\prime}\right)\right)=c\left(t, t^{\prime} ; \phi\right)=E\left(\left(g_{p}(t)-M(t)\right)\left(g_{p}\left(t^{\prime}\right)-M\left(t^{\prime}\right)\right)\right)
$$

where $\phi$ stands for the hyperparameters set. In a brief form, a Gaussian process $g_{p}(t)$ can be stated as in (3):

$$
g_{p}(t) \square G_{P}\left(M(t), c\left(t, t^{\prime}\right)\right.
$$


where $G_{P}$ denotes the Gaussian process. The brief presentation shows that the $g_{p}(t)$ function has a distribution forms the Gaussian process with kernel (covariance) function $c\left(t, t^{\prime}\right)$ and mean $M(t)$ [26, 33].

The aim of a regression problem is to form the dependence of a target $d$ on predictor variables $t_{i}$. Each target variable $d$ could be connected to a fundemantal arbitrary regression function $g_{p}(t)$ with an additive Gaussian noise $(\varepsilon)$ which is identically distributed and states the noise value from the data distribution. The mathmematical operation can be expressed as:

$$
d=g_{p}(t)+\varepsilon
$$

here, $\varepsilon$ the noise has zero mean value and its variance is $\sigma_{n}^{2}$. So we can rewrite the noise as $\varepsilon \square N\left(0, \sigma_{n}^{2}\right)$. Then, the Gaussian process shown in the (3) expands as in (5) [26]:

$$
g_{p}(t) \square G_{P}\left(M(t), c\left(t, t^{\prime}\right)+\sigma_{n}^{2} I\right)
$$

where, $I$ is for the identification matrix. The additive behave of the noise $\varepsilon$ and the marginalization feature of the $G_{P}$ forms a joint distribution belongs to output of training process $d$ and with locations of $T$ and outputs of test process $f_{*}$ with test points of $T_{*}$, the mathematical form of this joint distribution can be given as in (6) [26]:

$$
\left[\begin{array}{l}
d \\
f_{*}
\end{array}\right] \square N\left(\left[\begin{array}{l}
M(T) \\
M\left(T_{*}\right)
\end{array}\right],\left[\begin{array}{cc}
c\left(T, T+\sigma_{n}^{2} I\right) & c\left(T, T_{*}\right) \\
c\left(T_{*}, T\right) & c\left(T_{*}, T_{*}\right)
\end{array}\right]\right)
$$

On the conditions of Gaussian prior distribution with $T, d$, and $T_{*}$, the prediction distribution can be given as in (7):

$$
p\left(d_{*} \mid T, d, T_{*}\right) \square N\left(\bar{d}_{*}, \operatorname{var}\left(d_{*}\right)\right)
$$

where, $\bar{d}_{*}(8)$ is the prediction mean and $\operatorname{var}\left(d_{*}\right)(9)$ is the predicton variance [26]:

$$
\begin{gathered}
\bar{d}_{*}=M\left(T_{*}\right)+c\left(T_{*}, T\right)\left[c(T, T)+\sigma_{n}^{2} I\right]^{-1}(d-M(T)) \\
\operatorname{var}\left(d_{*}\right)=c\left(T_{*}, T_{*}\right)\left[c(T, T)+\sigma_{n}^{2} I\right]^{-1} c\left(T, T_{*}\right)
\end{gathered}
$$

In the GPR model covariance (kernel) function has a key role [26, 33]. Hence, on the way of the determination of the layout function, the selection of a proper covariance function is a crucial task. As a brief explanation, covariance function computes a similarity measure i.e., the inputs which are closely related to each other have similar targets; in parallel, test inputs neighboring a training input should be addressed to a corresponding target value neighboring the training input. In this manner, a test input could be predicted. There are many kinds of the covariance functions proposed in the literature. Some of those can be listed: Matern class, Gaussian noise, Ornstein - Uhlenbeck, Gama exponential, squared exponential, periodic, rational quadratic and so on [26, 33]. 


\section{Data Description}

In the proposed study, a GPR based ML model is designed for prediction of the surface roughness of the $\mathrm{Al}-\mathrm{SiC}_{\mathrm{p}}\left(\mathrm{LM} 25 / \mathrm{SiC} / 4_{\mathrm{p}}\right) \mathrm{MMC}$ with the cylindrical grinding experimental data. The cylindirical grinding workbench uses an aluminum oxide grinding wheel which is vitrified-bonded. The grinding wheel is capable of different speed levels because a variable speed drive is used with the wheel motor. Variable speed drive controls the frequency of the electrical supply of the wheel motor to obtain different speed levels of the grinding process. The real-world experimental dataset is collected from studies [3, 29] where the dataset information and details are shared publicly.

The inputs of the dataset is considered as 1) Velocity of the grinding wheel, $\left.V_{s}(\mathrm{~m} / \mathrm{min}), 2\right)$ Velocity of the work piece, $\left.V_{w}(\mathrm{~m} / \mathrm{min}), 3\right)$ Feed, $f(\mathrm{~m} / \mathrm{min})$, and 4) Depth of cut, $d$ $(\mu \mathrm{m})$. The output (target) parameter of the model is surface roughness, $R_{a}(\mu \mathrm{m})$. Measurement of the surface roughness is performed by the help of various methods, among them, the center line average (CLA) method is mostly preferred in the surface roughness measurement. CLA calculates the average deviation for the measurement from the nominal surface. Its mathematical expression is given in (10):

$$
R_{a}=\frac{1}{L} \int_{0}^{L} Y(x) d x
$$

where, $R_{a}$ states the arithmetic average deviation away from the mean line, $L$ is for the length of sampling, and $Y$ stands for the roughness profile ordinate. In the preferred dataset, the measurement of the surface roughness is performed with the CLA method [3]. By using the aforementioned dataset, our model opens the possibility to improve the machining process by surface roughness prediction. Table 1 lists the dataset properties with their feature names, definitions, symbols, and units.

Table 1. Dataset brief.

\begin{tabular}{llc}
\hline Feature & Definition & Symbol and Unit \\
\hline Input-1 & Velocity of the grinding wheel & $V_{s}(\mathrm{~m} / \mathrm{min})$ \\
Input-2 & Velocity of the work piece & $V_{w}(\mathrm{~m} / \mathrm{min})$ \\
Input-3 & Feed & $f(\mathrm{~m} / \mathrm{min})$ \\
Input-4 & Depth of cut & $d(\mu \mathrm{m})$ \\
Output & Surface roughness & $R_{a}(\mu \mathrm{m})$ \\
\hline
\end{tabular}

In order to perform a detailed visual experience about the preferred dataset, Figure 3 illustrates the dataset distribution. Here, all the input parameters can be seen with its distribution levels corresponding to output values. Every input parameter with each sample is demonstrated. As it is seen in Figure 3, all input parameters have three-level distribution. Figure 3 also draws the big picture of the preferred dataset by containing every sample with their output (target) values which is absolutely the surface roughness.

The details of the dataset can be found in the study [3], and the other details of real-world grinding experimental workbench can be reached with the study [29]. 


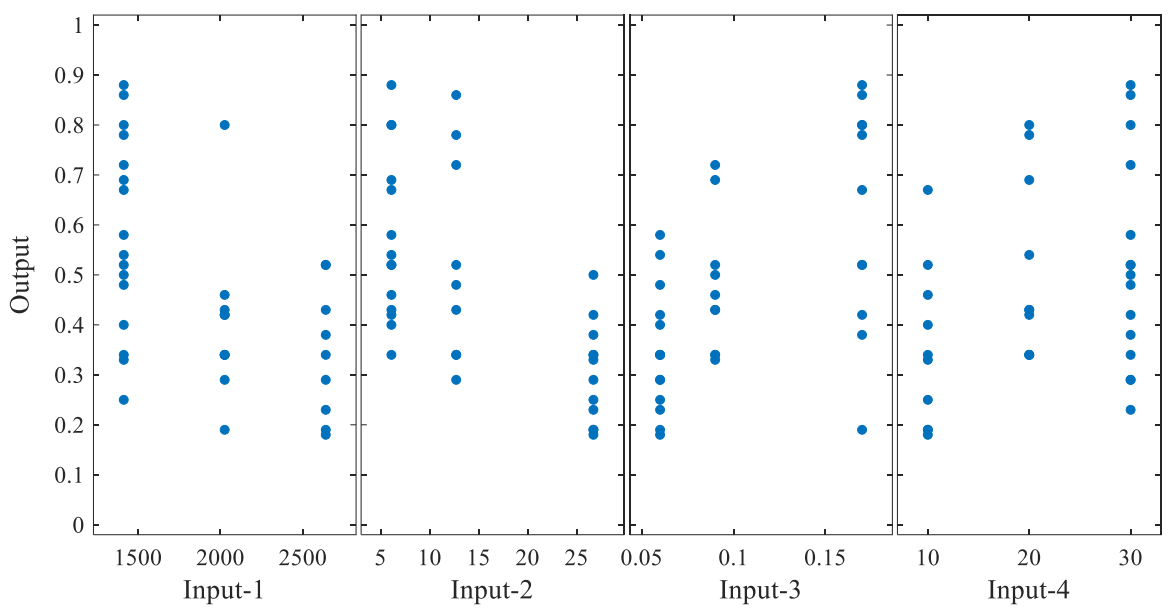

Figure 3. Dataset distribution.

\section{Setup and Experimental Findings}

In this section, we declare the experimental design of the proposed ML system for surface roughness prediction. Also, the evaluation metrics are presented. Lastly, this section contains the experimental findings of the proposed system. The effect of the proposed system on its prediction rates and other evaluation metrics are discussed. The experiments of the artificial intelligent based prediction system with its development codes are designed in MATLAB environment set on a workstation with Intel Xeon E5 dual-processor of $2.1 \mathrm{GHz}$, Quadro M4000 8GB of GPU memory, and 32 GB of RAM memory.

\subsection{Setup}

The details of the experiments are presented in this subsection. To obtain a thorough analysis, we evaluate the proposed model in challenging conditions. After the data preprocessing (normalization of the input parameters), the proposed GPR model (including hyperparameter optimization) is evaluated with two methods as data splitting ( $80 \%$ train set $-20 \%$ test set), and 5 -fold cross-validation. In the dataset normalization (just for input parameters not the targets), we process the data matrix by mapping its row minimum and maximum values to the interval of $[-1,1]$ with the help of "mapminmax" function.

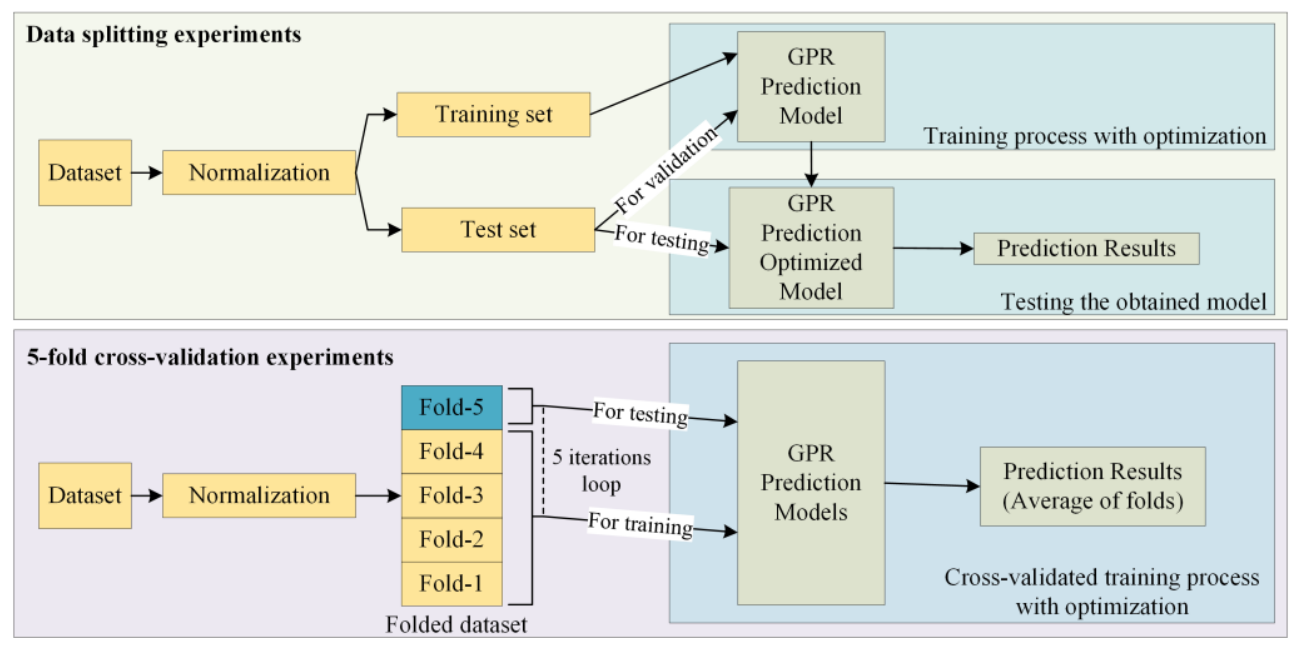

Figure 4. Experiment design of the proposed machine learning model. 
Figure 4 illustrates the block diagram of the experiment scenario of the proposed machine learning-based prediction model of GPR. In data splitting experiments, the best tuned prediction model is achieved in the training process with optimization. The optimization process tracks the validation error using the test set. After determining the best model, it is tested by the separate test set.

Table 2. Optimized hyperparameters of the GPR model.

\begin{tabular}{llcc}
\hline \multirow{2}{*}{ Hyperparameter } & Optimization interval & \multicolumn{2}{c}{ Specified Values of the Models } \\
\cline { 3 - 4 } & & Data Splitting & 5-fold \\
\hline Basis Function & Constant, zero, linear & Zero & Constant \\
Kernel & $\begin{array}{l}\text { Exp, Matern 5/2, Matern 3/2, } \\
\text { Isotropic, Rational quadratic }\end{array}$ & Matern 3/2 & Matern 5/2 \\
Kernel Scale & $(0.00240,2,4007)$ & 0.0064 & 0.7175 \\
Sigma & $(0.0001,1.9909)$ & 0.0164 & 0.4022 \\
Standardize & True, false & True & False \\
\hline
\end{tabular}

In Table 2, the hyperparameter details in the practice of the machine learning-based GPR model are listed. Here, we can see the optimization interval and specified values according to the experiment type. The basic hyperparameters as the kernel function type, kernel scale, basis function, sigma value, and the option of standardizing are included in the Bayesian optimization approach. In addition to the normalization, the standardizing process may improve performance in some situations. The optimization process tracks the validation error for 30 iterations.
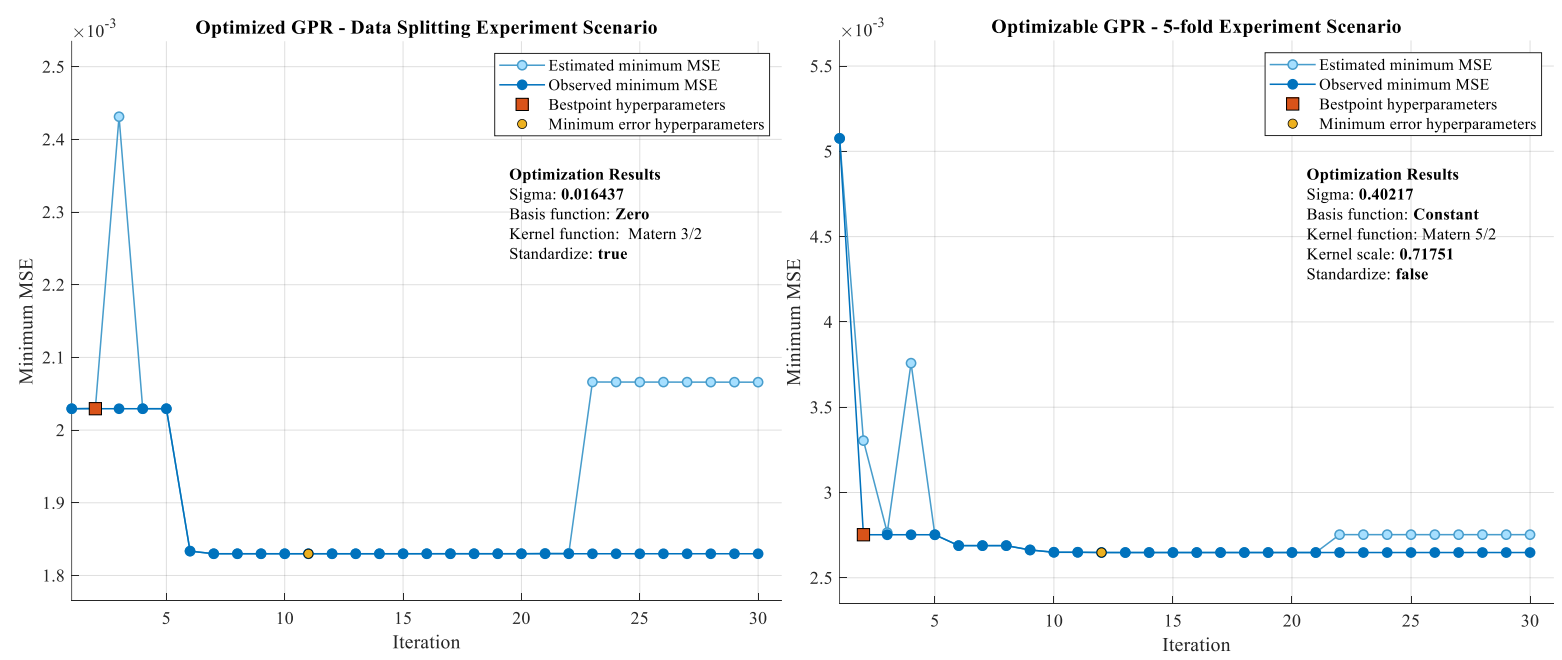

Figure 5. Prediction vs Actual plot and Response plot of the GPR model.

Figure 5 shows the optimization minimum objective tracing graphics of both two experiment scenarios as data splitting and 5-fold cross-validation. As we can see in both scenario's validation error tracing, the 5-fold cross-validation experiment provides a robust operation in optimization. In Figure 5, alongside the specified values, the best point hyperparameters and minimum error hyperparameters locations are also presented. 
To achieve an efficient evaluation of the performance values of the proposed machine learning based GPR model for surface roughness prediction, we prefer four well-known statistical criteria namely: mean absolute error (MAE), root mean square error (RMSE), mean square error (MSE), coefficient of determination (R-squared). The mathematical expressions of the evaluation criteria is given in (11)-(14):

$$
\begin{gathered}
\mathrm{MSE}=\frac{\sum_{i=1}^{N}\left(Y_{i}-\hat{Y}_{i}\right)^{2}}{N} \\
\mathrm{RMSE}=\sqrt{\mathrm{MSE}}=\sqrt{\frac{\sum_{i=1}^{N}\left(Y_{i}-\hat{Y}_{i}\right)^{2}}{N}} \\
\mathrm{MAE}=\frac{\sum_{i=1}^{N}\left|Y_{i}-\hat{Y}_{i}\right|}{N} \\
\text { R-squared }=1-\frac{\sum_{i=1}^{N}\left(Y_{i}-\hat{Y}_{i}\right)^{2}}{\sum_{i=1}^{N}\left(Y_{i}-\bar{Y}_{i}\right)^{2}}
\end{gathered}
$$

where, $Y_{i}$ is the actual values of the test dataset, $\hat{Y}_{i}$ is the predicted output values of the GPR model, $N$ is the total number of observations, and $\bar{Y}_{i}$ is the mean value of the actual test values.

\subsection{Experimental Findings}

In this subsection, we present the obtained results of the proposed GPR model with related graphical presentations and tables. For an efficient evaluation, quadratic and Gaussian SVM model results are also achieved for data splitting and 5-fold experiments. Table 3 shows the GPR model prediction results for both two experiment scenarios with prediction speed (observation per second) and trainin time (second).

Table 3. GPR prediction results.

\begin{tabular}{lcc}
\hline \multirow{2}{*}{ Criterion } & \multicolumn{2}{c}{ Performance } \\
\cline { 2 - 3 } & Data Splitting & 5-fold \\
\hline RMSE & 0.0454 & 0.0525 \\
R-squared & 0.98 & 0.93 \\
MSE & 0.0021 & 0.0027 \\
MAE & 0.0387 & 0.0397 \\
Prediction speed & $\sim 2100 \mathrm{obs} / \mathrm{sec}$ & $\sim 4000 \mathrm{obs} / \mathrm{sec}$ \\
Training time & $49.775 \mathrm{sec}$ & $49.266 \mathrm{sec}$ \\
\hline
\end{tabular}


In Table 3, we can see the detailed performance metrics of data splitting and 5-fold crossvalidation experimental results. In terms of RMSE evaluation, both models produce close values with each other as 0.0454 and 0.0525 , for data splitting and 5-fold scenarios. RMSE value of 5-fold experiments stays behind the data splitting because it is the challenging experiment for the model. But the robustness of the 5-fold experiments is proven with the MSE and MAE values even if its R-squared value is left behind of the data splitting. If it is analyzed the prediction speed and training time, both model provides an efficient computational cost. The prediction speed of the 5-fold scenario is faster because it operates smaller data packages according to the data splitting method. The training times are given for 30 iterations of the optimization process, so we can assume that a one-time run of both models takes approximately 1.5 seconds for prediction.

To provide a visual experience of the model predictions, we present a collection of graphical representations of the experimental findings of the proposed prediction model. Figure 6 illustrates the plot of predicted vs actuals of the predicted values. Both experiment scenario performs well that predictions are close to the perfect prediction line. In Figure 7, we can see a detailed presentation of the predicted and actual values as a response plot. Here, we can see the prediction errors, clearly.
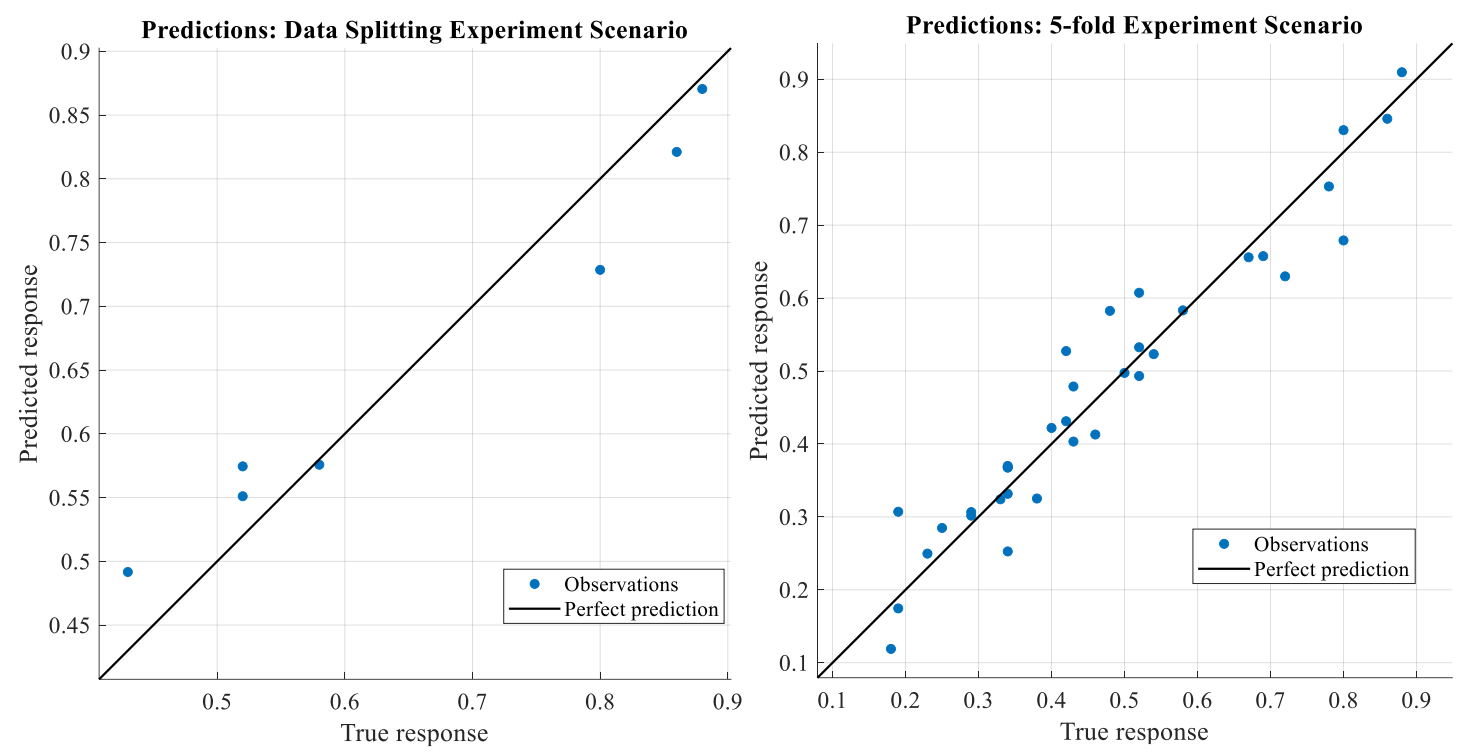

Figure 6. Prediction vs Actual plots of the GPR models for two experiment scenarios.
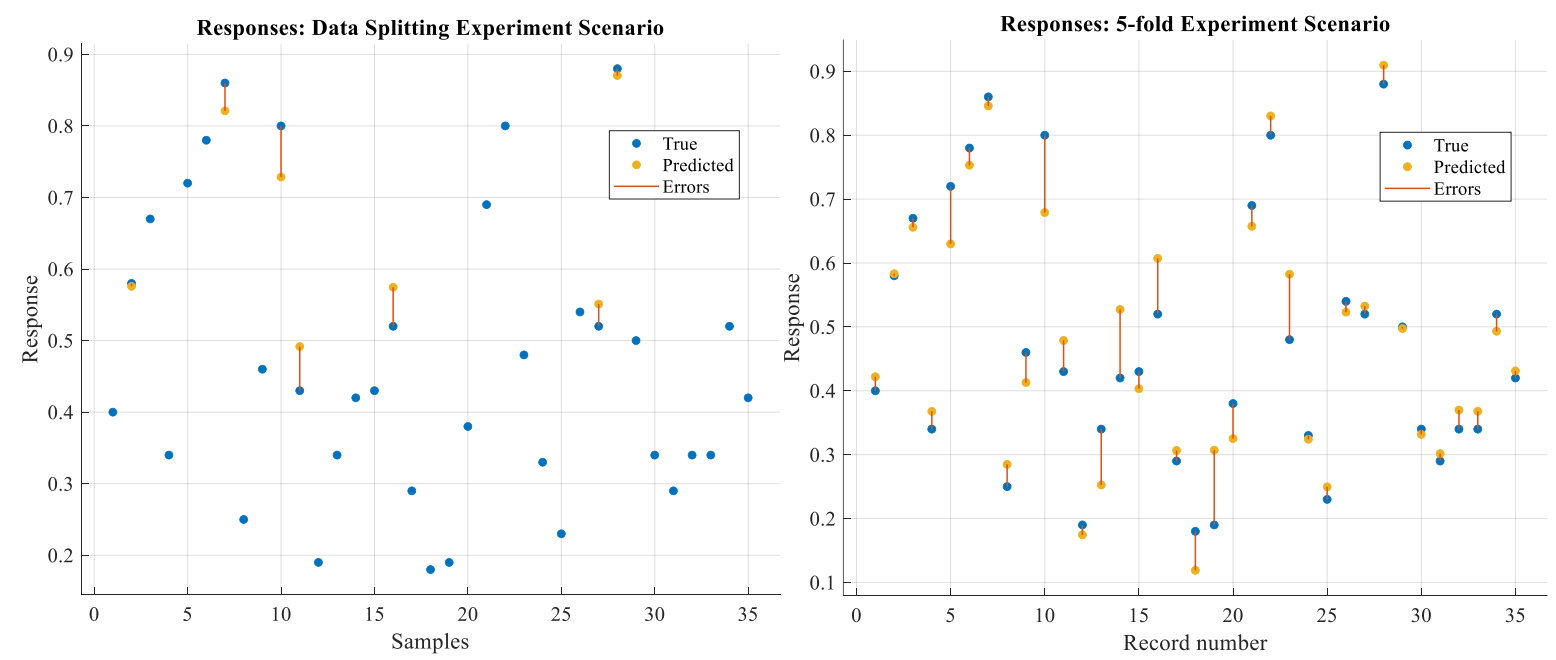

Figure 7. Response plots of the GPR models for two experiment scenarios. 

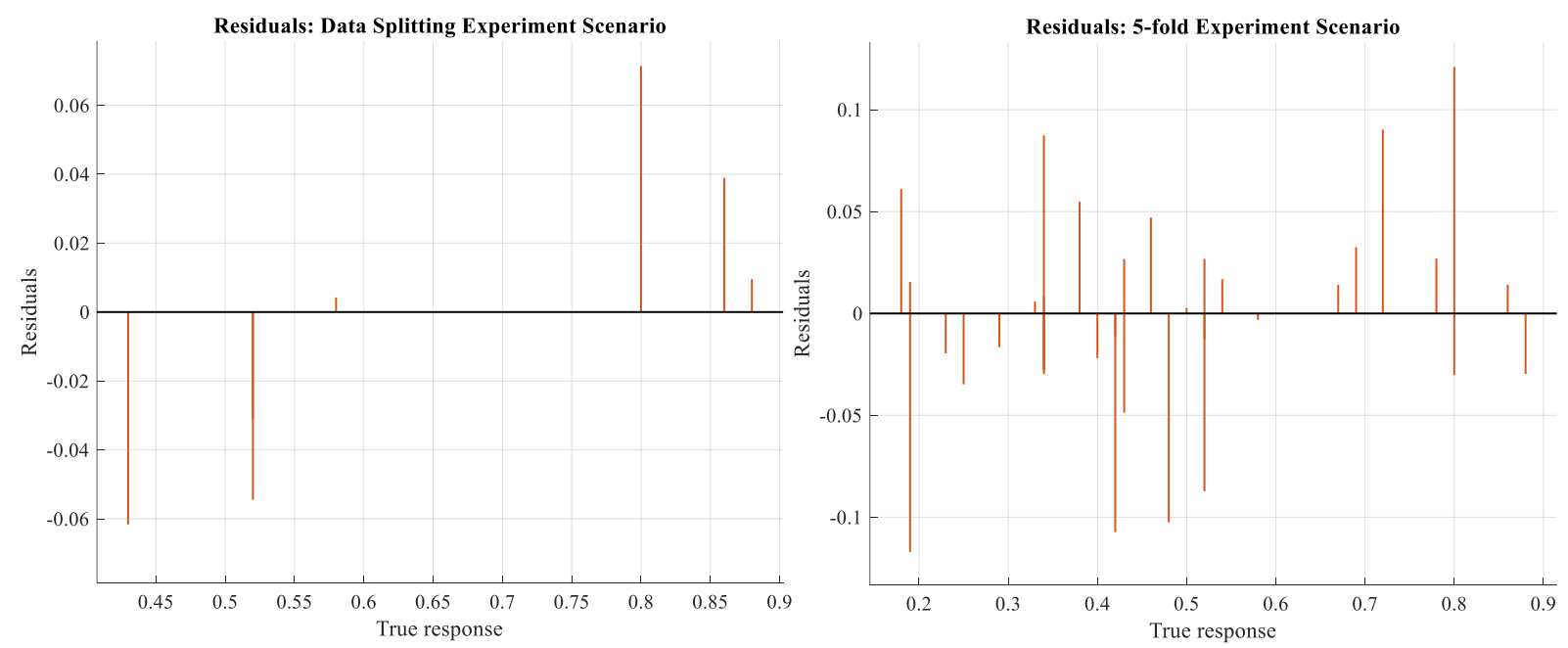

Figure 8. Residuals of the errors in the GPR models for two experiment scenarios.

In Figure 8, residuals of the errors in the GPR models are presented for two experiment scenarios. Here, we can see the distances of the errors to the zero line. Figure 8 clearly shows the error rate of the each instance of the test dataset.

Table 4. GPR model prediction results comparison with SVM models

\begin{tabular}{lcccc}
\hline Criterion & \multicolumn{1}{c}{ Proposed Optimized GPR Model } & \multicolumn{2}{c}{ Optimized SVM Models } \\
\cline { 2 - 5 } & Data Splitting & 5-fold & $\begin{array}{c}\text { Data Splitting } \\
\text { (quadratic) }\end{array}$ & $\begin{array}{c}\text { 5-fold } \\
\text { (Gaussian) }\end{array}$ \\
\hline RMSE & 0.0454 & 0.0525 & 0.0795 & 0.0553 \\
R-squared & 0.98 & 0.93 & 0.81 & 0.92 \\
MSE & 0.0021 & 0.0027 & 0.0063 & 0.0031 \\
MAE & 0.0387 & 0.0397 & 0.0575 & 0.0455 \\
Prediction speed & $\sim 2100 \mathrm{obs} / \mathrm{sec}$ & $\sim 4000 \mathrm{obs} / \mathrm{sec}$ & $\sim 2900 \mathrm{obs} / \mathrm{sec}$ & $\sim 1900 \mathrm{obs} / \mathrm{sec}$ \\
Training time & $49.775 \mathrm{sec}$ & $49.266 \mathrm{sec}$ & $73.453 \mathrm{sec}$ & $85.053 \mathrm{sec}$ \\
\hline
\end{tabular}

In Table 4, we provide a complete comparison between the proposed optimized GPR model and optimized SVM models. It can be interpreted from Table 4 that the SVM models stay behind the proposed GPR model in all experiment scenarios. The highest performance of SVM models is achieved in the scenario of 5-fold experiment with an RMSE value of 0.553 but considering the other performance metrics, proposed GPR model outperforms the SVM which is one of the well-known benchmark method in machine learning.

Although there would be no fair comparison due to the structural dynamics of the ANN method and its many adjustable parameters, we provide a comparison table including the results of the study [3]. Table 5 presents a comparison between the proposed machine learning based GPR prediction model and traditional ANN model of study [3]. As mentioned above, the study [3] uses the same dataset with the proposed study. But, the authors organize a three-part dataset as 20 samples for training, 5 samples for testing, and 10 samples for validation, although there is no optimization process. The authors of the study [3] do not provide the same performance metrics, so we transform their validation results table to RMSE and MSE form and build the Table 5. As it is seen in Table 5, the proposed GPR model catches 
the traditional ANN with close RMSE and MSE values. Also, the proposed GPR model includes few parameters according to the ANN method with lots of dynamic parameters. The proposed surface roughness prediction model is computationally efficient with its machine learning basis comparing to traditional ANN.

Table 5. GPR prediction results: Comparsion with ANN

\begin{tabular}{llc}
\hline \multirow{2}{*}{ Models } & \multicolumn{2}{c}{ Performance } \\
\cline { 2 - 3 } & RMSE & MSE \\
\hline Proposed GPR Model & 0.0454 & 0.0021 \\
ANN Model [3] & 0.0301 & 0.0010 \\
\hline
\end{tabular}

\section{Conclusion}

In this article, a novel approach for the prediction of the surface roughness of the Al-based MMC in cylindrical grinding has been successfully applied. An optimized GPR model is designed with two different experimental scenarios using a real-life dataset. For this prediction modeling, the experimental dataset is achieved from the studies [3,29]. The workpiece velocity, the wheel velocity, depth of cut, and feed are considered as input parameters of the model while the surface roughness as the output of the model. The performance metrics of the proposed machine learning-based GPR model are thought to be acceptable. The proposed machine learning-based GPR model with its easy to implement structure and prediction ability is a promising method to improve the machining quality of the Al MMC materials in analyzing the surface roughness. The proposed model predicts the surface roughness successfully with 0.98 RMSE value in challenging experiments. Considering the two different scenarios applied, the 5-fold cross-validation experiment scenario turns out to be more robust. Although the R-squared value of this scenario stays behind the data splitting, MAE and RMSE values prove the impact of the proposed model on surface roughness prediction. In the proposed GPR model, the hyperparameter optimization is also applied using the essentials of the Bayesian approach. Thus, the most important parameters of the GPR method such as kernel function type and sigma value are designed in the optimized model. Based on obtained results, it is concluded that the prediction model in this study could be to help the related experts to provide low-cost experiments. In addition, the proposed GPR model could be effectively utilized for the prediction modeling of different machining processes of various material components after re-training it with the related dataset. For the further studies, different optimization algorithms with metaheuristic methods could be explored to improve the prediction ability of the GPR model.

\section{References}

[1] Devarasiddappa, D., et al. (2012). Artificial neural network modeling for predicting surface roughness in end milling of $\mathrm{Al}-\mathrm{SiCp}$ metal matrix composites and its evaluation. Journal of Applied Sciences 12, 955-962.

[2] Laghari, R.A, et al. (2019). A review on application of soft computing techniques in machining of particle reinforcement metal matrix composites. Archives of Computational Methods in Engineering, 1-15. 
[3] Chandrasekaran, M., Devarasiddappa, D. (2014). Artificial neural network modeling for surface roughness prediction in cylindrical grinding of $\mathrm{Al}-\mathrm{SiC} \mathrm{p}$ metal matrix composites and ANOVA analysis. Advances in Production Engineering \& Management, 9, 59-70.

[4] Yakut, R., Ürkmez Taşkın, N. (2019). Production of AA7075/B 4 C composite materials by semisolid stirring method. European Journal of Technique, 9, 230-240.

[5] Ürkmez Taşkın, N., et al. (2020). The effects of welding pressure and reinforcement ratio on welding strength in diffusion-bonded $\mathrm{AlMg}_{3} / \mathrm{SiCp}$ Composites. European Journal of Technique, 10,75-85.

[6] Conduit, B.D., et al. (2017). Design of a nickel-base superalloy using a neural network. Materials and Design, 131, 358-365.

[7] Chen, C-T, Gu, G.X. (2019). Machine learning for composite materials. MRS Communications, $9,556-566$.

[8] Agrawal, A., Choudhary, A. (2018). An online tool for predicting fatigue strength of steel alloys based on ensemble data mining. International Journal of Fatigue, 113, 389-400.

[9] Bock, F.E., et al. (2019). A review of the application of machine learning and data mining approaches in continuum materials mechanics. Frontiers in Materials, 6, 110.

[10] Yang, C., et al. (2020). Prediction of composite microstructure stress-strain curves using convolutional neural networks. Materials and Design, 189, 108509.

[11] Sizemore, N.E., et al. (2020). Application of machine learning to the prediction of surface roughness in diamond machining. Procedia Manufacturing, 48, 1029-1040.

[12] Ajith Arul Daniel, S., et al. (2019). Multi objective prediction and optimization of control parameters in the milling of aluminium hybrid metal matrix composites using ANN and Taguchi -grey relational analysis. Defence Technology, 15, 545-556.

[13] Phate, M.R., Toney, S.B. (2019). Modeling and prediction of WEDM performance parameters for $\mathrm{Al} / \mathrm{SiCp} \mathrm{MMC}$ using dimensional analysis and artificial neural network. Engineering Science and Technology, an International Journal, 22, 468-476.

[14] Babalola, P.O., et al. (2017). Artificial neural network prediction of aluminium metal matrix composite with silicon carbide particles developed using stir casting method. International Journal of Mechanical \& Mechatronics Engineering, 15 (6), 151-159.

[15] Amirjan, M., et al. (2013). Artificial neural network prediction of $\mathrm{Cu}-\mathrm{Al}_{2} \mathrm{O}_{3}$ composite properties prepared bypowder metallurgy method. Journal of Materials Research and Technology, 2 (4), 351-355.

[16] Varol, T., Ozsahin, S. (2019). Artificial neural network analysis of the effect of matrix size and milling time on the properties of flake $\mathrm{Al}-\mathrm{Cu}-\mathrm{Mg}$ alloy particles synthesized by ball milling. Particule Science and Technology, 37, 381-390.

[17] Ugrasen, G., et al. (2018). Estimation of machining performances using MRA and GMDH in Wire EDM of Al2024 based Hybrid MMC. Materilas Today: Proceedings, 5, 3084-3092. 
[18] Özyürek, D., et al. (2014). Experimental investigation and prediction of wear properties of $\mathrm{Al} / \mathrm{SiC}$ metal matrix composites produced by thixomoulding method using Artificial Neural Networks. Materials and Design, 63, 270-277.

[19] Chuanmin, Z., et al. (2019). Surface roughness prediction model of SiCp/Al composite in grinding. Internatıonal Journal of Mechanical Sciences, 155, 98-109.

[20] Zain, A.M., et al. (2010). Prediction of surface roughness in the end milling machining using Artificial Neural Network. Expert Systems with Applications, 37, 1755-1768.

[21] Kumar, R., Chauhan, S. (2015). Study on surface roughness measurement for turning of Al 7075/10/SiCp and Al 7075 hybrid composites by using response surface methodology (RSM) and artificial neural networking (ANN). Measurement: Journal of the International Measurement Confederation, 65, 166-180.

[22] Thankachan, T., et al. (2019). Prediction of surface roughness and material removal rate in wire electrical discharge machining on aluminum based alloys/composites using Taguchi coupled Grey Relational Analysis and Artificial Neural Networks. Applied Surface Science, $472,22-35$.

[23] Marani, M., et al. (2019). Neuro-fuzzy predictive model for surface roughness and cutting force of machined $\mathrm{Al}-20 \mathrm{Mg}_{2} \mathrm{Si}-2 \mathrm{Cu}$ metal matrix composite using additives. Neural Computing and Applications, 1-12.

[24] Prakash Rao, C.R., et al. (2014). Effect of machining parameters on the surface roughness while turning particulate composites. Procedia Engineering, 97, 421-431.

[25] Şahin, İ. (2014). Prediction of surface roughness of $\mathrm{Al} / \mathrm{SiC}$ composite material with artificial neural networks. Journal of Faculty of Engineering and Architecture of Gazi Univesity, 29, 209-216.

[26] Arthur, C.K., et al. (2020). Novel approach to predicting blast-induced ground vibration using Gaussian process regression. Engineering with Computers, 36, 29-42.

[27] Schmidt, J., et al. (2019). Recent advances and applications of machine learning in solid-state materials science. npj Computational Materials, 5, 83.

[28] Koçyiğit, F, et al. (2017). Prediction of thermal performance of designed different obstacles on absorber plates in solar air collectors by support vector machine. European Journal of Technique, 7, 186-194.

[29] Thiagarajan, C., et al. (2011). Cylindrical grinding of SiC particles reinforced aluminium metal matrix composites. ARPN Journal of Engineering and Applied Scieces, 6, 14-20.

[30] Gurgenc, T., et al. (2019). A study on the extreme learning machine based prediction of machining times of the cycloidal gears in CNC milling machines. Production Engineering, 13, 635-647.

[31] Ucar, F., Korkmaz, D. (2020). COVIDiagnosis-Net: Deep Bayes-Squeeze Net based diagnosis of the coronavirus disease 2019 (COVID-19) from X-ray images. Medical Hypotheses, 140,109761. 
[32] Ekici, S., et al. (2020). Power quality event classification using optimized Bayesian convolutional neural networks. Electrical Engineering. (Early published)

[33] Rasmussen, C.E., Williams, C.K.I. Gaussian processes for machine learning. MA:MIT Press, Cambridge, Massachusetts, 2006. 